\title{
L'adversaire de Dieu - Der Widersacher Gottes
}

\author{
6. Symposium Strasbourg, Tübingen, Uppsala. 27.-29. Juni 2013 in Tübingen \\ Hrsg. v. Michael Tilly, Matthias Morgenstern u. Volker Henning Drecoll unter \\ Mitarb. v. Hendrik Stoppel
}

[The Adversary of God. The Sixth Strasbourg-Tübingen-Uppsala Symposium. Tübingen, 27-29 June 2013.]

L'adversaire de Dieu Der Widersacher Gottes

$$
\begin{aligned}
& \text { Herausgegeben von } \\
& \text { MICHAEL TILLY, }
\end{aligned}
$$
MATTHIAS MORGENSTERN und VOLKER HENNING DRECOLL



2016. XIV, 359 Seiten. WUNT I 364

SBN 978-3-16-154237-4

DOI 10.1628/978-3-16-154237-4 eBook PDF $159,00 €$

ISBN 978-3-16-154236-7

Leinen $159,00 €$
Veröffentlicht auf Französisch.

This trilingual collection contains studies on how the literary guises of God's enemies originated, how they were presented and what meaning they held in the Jewish Holy Scriptures, the Christian Bible, ancient and rabbinical Jewish writings, in early Christianity and in gnostic texts. Exegetic-philological, history of religion, Jewish- as well as conceptual and history of theology aspects are all comprehensively dealt with. Scholars from the universities of Strasbourg, Tübingen and Uppsala's evangelical theology faculties examine Satan, Beelzebub, the Anti-Christ, the diabolical, demons, evil intent and other God-opposing forces, as well as the Danish filmmaker Lars von Trier's treatment of evil, in their contributions.

\section{Contributors:}

Gabriella Aragione, Volker Henning Drecoll, Göran Eidevall, Tord Fornberg, Christian Grappe, Gabriella Gustafsson, Gudrun Holtz, Jan Joosten, James A. Kelhoffer, Mikael Larsson, Martin Leuenberger, Hermann Lichtenberger, Matthias Morgenstern, Anna-Maria Schwemer, Michael Theobald, Madeleine Wieger

Inhaltsübersicht

Martin Leuenberger: Widersacher-Konstellationen in der Levante und im AT - Göran Eidevall: The Role of the Enemies of Yhwh in the Book of Isaiah and in the Psalms - Jan Joosten: „Pour mettre fin à l'ennemi et au vindicatif«. La thématique du Psaume 8 - Hermann Lichtenberger: Der Feind Gottes in der frühjüdischen Literatur - Antiochus IV Epiphanes - Christian Grappe: Éclairage sur les récits du baptême de Jésus, de son épreuve au désert et de sa victoire sur Satan à partir surtout de la littérature intertestamentaire - Tord Fornberg: Baal-zevul - The History of a Name - Gudrun Holtz: Die Zeit der Verkündigung des Reiches Gottes und das Wirken des Widersachers im lukanischen Doppelwerk - James A. Kelhoffer: Der Verfolger als Gottes Widersacher in der Apostelgeschichte - Anna-Maria Schwemer: Agrippa I. - Sein Tod als »Gottesfeind « bei Josephus und Lukas - Michael Theobald: Der Widersacher im Johannesevangelium - Madeleine Wieger: „Celui qu'on appelle

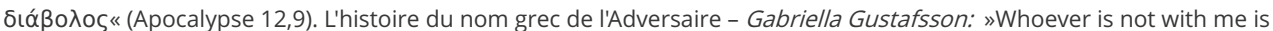
against me«. Accounts of Rituals Securing the Loyalty of Individual Gods in Republican Rome - Matthias Morgenstern: Auf der Suche nach der »anderen Seite« Gottes in der rabbinischen Literatur - Erwägungen zu einigen Stellen im Babylonischen Talmud - Gabriella Aragione: L'ange premier-né et les theomachoi dans l'écrit Aux Grecs de Tatien - Volker Henning Drecoll: Saklas - Mikael Larsson: Whose Enemy? Lars von Trier's Anti-Christ in Dialogue with Biblical Creation and Passion Narratives

Michael Tilly Geboren 1963; Studium der Ev. Theologie in Mainz und Heidelberg; 1993 Promotion und 2001 Habilitation an der Johannes Gutenberg-Universität Mainz; Professor für Neues Testament an der Eberhard Karls Universität Tübingen. https://orcid.org/0000-0002-7217-3782

Matthias Morgenstern Geboren 1959; Studium der Ev. Theologie und der Judaistik; 1995 Promotion; 2000 Habilitation; apl. Professor am Seminar für Religionswissenschaft und Judaistik/Institutum Judaicum der Universität Tübingen, Ev.-Theologische Fakultät.

https://orcid.org/0000-0001-7569-2273

Volker Henning Drecoll Geboren 1968; Studium der Ev. Theologie in Münster; 1996 Promotion; 1998 Habilitation; seit 2004 Professor für Kirchengeschichte mit dem Schwerpunkt Alte Kirche in Tübingen; seit 2005 Ephorus des Evangelischen Stifts. https://orcid.org/0000-0001-7641-4662

Hendrik Stoppel ist Wissenschaftlicher Mitarbeiter an der Forschungsstätte der Evangelischen Studiengemeinschaft in Heidelberg.

https://orcid.org/0000-0002-7404-1679

Jetzt bestellen:

https://mohrsiebeck.com/buch/ladversaire-de-dieu-der-widersacher-gottes-9783161542374?no_cache=1 order@mohrsiebeck.com

Telefon: +49 (0)7071-923-17

Telefax: $+49(0) 7071-51104$ 